\title{
Self-Perceived Health of Parents of Children with Autism Spectrum Disorders: Relation with the Severity Level
}

\author{
Clarisse Chatel Garriot' ${ }^{1}$, Virginie Villes ${ }^{2}$, Anne Marie Bartolini' ${ }^{1}$, François Poinso ${ }^{1}$ \\ ${ }^{1}$ Autism Resource Center, University Hospital Marseille, Sainte Marguerite Hospital, Marseille, France \\ ${ }^{2}$ Department of Public Health, University Hospital Marseille, Marseille, France \\ Email: Clarisse.chatel@ap-hm.fr
}

Received 4 October 2014; revised 2 November 2014; accepted 26 November 2014

Copyright @ 2014 by authors and Scientific Research Publishing Inc.

This work is licensed under the Creative Commons Attribution International License (CC BY). http://creativecommons.org/licenses/by/4.0/

(c) (i) Open Access

\section{Abstract}

Children with autism spectrum disorders (ASD) are characterized by deficits in reciprocal social interaction and communication which have a major effect on the quality of life of the parents. Self-perceived health of 124 ASD parents was investigated using the short-form health survey (SF36) questionnaire. In a first step, comparative analysis was performed between a control group of parents and three groups of ASD parents classified according to the severity levels for autism spectrum disorder. In a second step, correlation analysis was performed between the self-perceived health of ASD parents and a continuous rating scale of the severity level of ASD children, determined with the Childhood Autistic Rating Scale (CARS) and the Autism Diagnostic Observation Schedule-Generic (ADOS-G). Results show that as the severity level of ASD symptoms increase, mother's perception of their own health decreases. Statistical analysis revealed a very strong relationship between the Mental Health of the mothers and the ASD children, especially with the deficit in social interaction. Restrictive and repetitive behavior and difficulty in play have less direct impact on the quality of life of ASD mothers. The highest impairment in quality of life was observed for the mothers of severe autism children, in the time spent at work and the difficulty to perform activities, due to mental and physical problems. Relationship between fathers and ASD children is significantly lower than that observed for mothers, except for problems with work or other daily activities as a result of physical health. These finding must be taken into account to provide better and more focused support to ASD mothers, in order to improve the mental health and to reduce the difficulty in performing activities.

\section{Keywords}

Autism Spectrum Disorders, Childhood Autistic Rating Scale, Parents, Self-Perceived Health 


\section{Introduction}

Autism spectrum disorders (ASD) are neurodevelopmental disorders present from early childhood and characterized by persistent impairment in reciprocal social communication and social interaction, and restricted, repetitive patterns of behaviors (American Psychiatric Association, 2013). These behaviours can range in impact from mild to disabling. ASD encompasses disorders previously referred to as childhood autism and Pervasive Developmental Disorder, Not Otherwise Specified (PDD-NOS).

Severity for children with moderate or severe autism is respectively based on marked deficits or severe deficits in social communication, in verbal and nonverbal communication, inflexibility of behaviors and narrow, obsessive interests. Pervasive Developmental Disorder, Not Otherwise Specified is used to refer to children who have significant problems in social communication and play, and some difficulties in interacting with others.

Social communication difficulties and restricted pattern of behavior have an impact on the quality of life of the parents. The World Health Organization define the quality of life as an individual's perception of their position in life in the context of the culture and value system in which they live, and in relation to their goals, expectations, standards and concern (WHOQOL Group, 1995: p. 1405).

Several studies on children with a disability revealed negative effect on the parents (Shu, 2009), especially stress (Beckman, 1995; Dyson, 1991), and depression (Abbeduto et al., 2004; Harvey et al., 1997; Trute, 1995; Zablotsky et al., 2012). Parents of ASD children are at risk for high levels of distress (Hastings, 2002). Fifty percent of mothers with children with autism symptoms had elevated depression scores compared to $15 \%$ - 21\% in the other groups (Olsson et al., 2001). The level of parental stress has been found to be related to the level of severity and disability of the children's diagnoses and to coexisting behavior problems (Freeman et al., 1991; Hastings, 2002).

It has been suggested that the decrease in quality of life is more pronounced for the ASD mothers than the fathers (Allik et al., 2006; Herring et al., 2006). Mothers reported that the severity of ASD child behaviors was significantly stressful (Freeman et al., 1991).

The aim of the present study was to compare the self-perceived health of parents with PDD-NOS children, parents with children with moderate and severe autism, with those of a control group of parent and to determine whether there was any difference between ASD mothers and ASD fathers. In addition, this study sought to examine the correlation between the severity level of ASD children and the self-perceived health of parents.

\section{Materials and Methods}

\subsection{Participants and Procedures}

The Autism Resource Center, Sainte Marguerite Hospital, Marseille, staff invited ASD parents to participate in the present study. Parents were approached when they came to the center and informed on the purpose of the study. The research was conducted with a multidisciplinary team; each member is specialized and experienced in working with ASD children.

Children were diagnosed according to DSM IV-TR criteria (American Psychiatric Association, 2000) and also Autism Diagnostic Interview-Revised (ADI-R) (Rutter et al., 2003) and Autism Diagnostic Observation Schedule-Generic (ADOS-G) (Lord et al., 2000). Each child has also been evaluated with cognitive measure (Wechsler, 1992; Wechsler, 2002).

\subsubsection{Characteristics of the ASD Children}

After functional evaluation and confirmation of the diagnosis of the children there remained 65 ASD children including 24 PDD-NOS children, 24 children with moderate autism and 17 children severe autism. The degree of autistic disorder was assessed by the Childhood Autistic Rating Scale (CARS) (Schopler et al., 1980, 1988; 2010). The CARS has been reported to have high validity to quantify the severity of ASD children (Coplan et al., 2004). The characteristics of the ASD children and the score on the CARS are reported in Table 1.

In the present study, we didn't have children with PDD-NOS with a score on the CARS higher than 30. All ASD children were free of psychotropic medication at the time of the study. The 65 ASD children were between 3 and 15 years of age at the time of the study, with a mean age of 6 years. 83\% of ASD children were male. Intellectual disability is a generalized disorder appearing in $12 \%$ of the children with PDD-NOS, half of the moderate autism children and all of the severe autism children. The diagnosis of intellectual disability take into account an IQ below 70 and significant limitations in two or more areas of adaptive behavior. 
Table 1. Characteristics of the ASD children.

\begin{tabular}{|c|c|c|c|c|}
\hline Characteristics & $\begin{array}{c}\text { Total ASD } \\
n=65\end{array}$ & $\begin{array}{c}\text { PDD-NOS } \\
n=24\end{array}$ & $\begin{array}{l}\text { Moderate Autism } \\
\qquad \mathrm{n}=24\end{array}$ & $\begin{array}{c}\text { Severe Autism } \\
\mathrm{n}=17\end{array}$ \\
\hline Age (mean \pm SD) & $6.3 \pm 2.8$ & $7.4 \pm 3.1$ & $6.3 \pm 2.9$ & $5.1 \pm 2.1$ \\
\hline Age 3 to 5 years (\%) & 46 & 39 & 50 & 55 \\
\hline Gender (male/female \%) & 83 & 83 & 83 & 82 \\
\hline CARS score $($ mean $\pm \mathrm{SD})$ & $32 \pm 7$ & $25 \pm 3$ & $33 \pm 2$ & $41 \pm 3$ \\
\hline ADOS Deficits in social interaction (mean \pm SD) & $9 \pm 4$ & $5 \pm 3$ & $10 \pm 3$ & $13 \pm 1$ \\
\hline ADOS Deficits in communication (mean \pm SD) & $5 \pm 3$ & $3 \pm 1$ & $6 \pm 3$ & $7 \pm 1$ \\
\hline Intellectual disability (\%) & 49 & 12 & 50 & 100 \\
\hline
\end{tabular}

\subsubsection{Parents of Children with ASD}

A total of 65 ASD mothers and 59 ASD fathers participate in the study. The 124 parents were divided into three groups, 44 parents of PDD-NOS children, 38 parents of children with moderate autism and 42 parents of children with severe autism. The mean ages \pm standard deviation of the mothers and the fathers were $39 \pm 7$ and $41 \pm$ 7 years respectively. $81 \%$ of the 65 families consisted of two-parent families. There was not a significant difference between the education levels of mothers and fathers, secondary education (60\%) and post-secondary education (40\%).

\subsubsection{Control Parents}

An additional sample of mothers $(n=23)$ and fathers $(n=22)$ of a typically developing child with a similar age distribution of study group, served as control parents and will hereafter be referred to as the control group. The 45 control parents were selected from primary and secondary schools located in the south of France. Aside from the age of the children, between 3 and 16 years, and the fact that they live in the south of France, no other demographic data for the control group was included.

\subsection{Scale and Measures}

\subsubsection{Self-Perceived Health of Parents Questionnaire (SF-36)}

Self-perceived health of parents was assessed using the health status questionnaire SF-36. The French version of the SF-36 (Leplège et al., 1998) has been transmitted to each of the 124 ASD parents and the 45 control parents. The mothers and fathers were asked to complete it independently of one another.

The SF-36 is a generic test and has been reported to have high validity and internal consistency (McHorney et al., 1993). Empirical studies suggest that the SF-36 provides a practical alternative to longer measures, and that the eight scales and two summary scales rarely miss a noteworthy difference in physical or mental health status in group level comparisons (Ware et al., 1994).

The eight scales are ordered in Table 2 in terms of their factor content. The first scale is Physical Functioning, which has been shown to be the best all around measure of physical health. The last scale, Mental Health is the most valid measure of mental health. Scales in between are ordered according to their validity in measuring physical and mental health. The mean score computed for the eight health domains ranged from 0 to 100 , with the high score indicating superior health.

Standard scoring algorithms were used for aggregating scores from the eight SF-36 scales in two distinct, higher-order summary scores, Physical Component Summary and Mental Component Summary. The scoring algorithms used for the summary scores take into account a mean of 50 and a standard deviation of 10, for a reference norm population.

\subsubsection{Childhood Autistic Rating Scale (CARS)}

The CARS is a behavior rating scale designed to help differentiate children with autism and to determine the severity of their autism. The CARS is a standardized clinical observation tool that rates 15 clinical features (social relatedness, stereotypical language, repetitious behaviors, sensory phenomena, etc.) from 1 (normal) through 4 (severely abnormal). Thus, scores on the CARS range from 15 (no signs of ASD) to 60 (highly severe ASD). Scores from 30 to 37 indicate moderate autism symptoms and from 37 to 60 severe autism symptoms. 
Table 2. Explanations of lowest and highest scores in the SF-36 scales.

\begin{tabular}{|c|c|c|}
\hline Scales & Lowest possible score & Highest possible score \\
\hline Physical Functioning & Very limited in performing all physical activities & $\begin{array}{l}\text { Performs all types of physical activities including the } \\
\text { most vigorous without limitations due to health }\end{array}$ \\
\hline Role-Physical & $\begin{array}{l}\text { Problems with work or other daily activities as a } \\
\text { result of physical health }\end{array}$ & No problems with work or other daily activities \\
\hline Bodily Pain & Very severe and extremely limiting pain & Feels peaceful, happy, and calm all of the time \\
\hline General Health & $\begin{array}{l}\text { Evaluates personal health as poor and believes it is } \\
\text { likely to get worse }\end{array}$ & Evaluates personal health as excellent \\
\hline Vitality & Feels tired and worn out all of the time & Feels full of pep and energy all of the time \\
\hline Social Functioning & $\begin{array}{l}\text { Extreme and frequent interference with normal social } \\
\text { activities due to physical and emotional problems }\end{array}$ & $\begin{array}{l}\text { Performs normal social activities without interference } \\
\text { due to physical or emotional problems }\end{array}$ \\
\hline Role-Emotional & $\begin{array}{l}\text { Problems with work or other daily activities as a } \\
\text { result of emotional problems }\end{array}$ & No problems with work or other daily activities \\
\hline Mental Health & Feelings of nervousness and depression all of the time & Feels peaceful, happy, and calm all of the time \\
\hline
\end{tabular}

Several studies indicated high concordance between the CARS and clinical diagnosis using DSM-IV criteria for autistic disorder (Perry et al., 2005; Trute, 1995). Studies on children reported a sensitivity of 0.98 when using the CARS for diagnoses of autistic disorder, with a CARS score at or above the autism cutoff of 30 (Eaves et al., 1993). Several studies report high internal consistency, with acceptable Cronbach's alpha coefficient $\geq 0.90$ (Magyar et al., 2007; Schopler et al., 1988). These results suggest that the CARS is a valid and reliable instrument for evaluating the presence and severity of symptoms of autism spectrum disorders.

\subsubsection{Autism Diagnostic Observation Schedule-Generic (ADOS-G)}

The Autism Diagnostic Observation Schedule-Generic (ADOS-G) is a semistructured assessment of social interaction and communication for children who may have autism or other pervasive developmental disorders. The protocol consisted of a series of structured and semi-structured tasks between the examiner and the subject. The scores for social interaction range from 0 to 14 (highly severity level). Scores for communication range from 0 to 10 .

\subsubsection{Statistical Analysis}

The Cronbach's alpha levels (Cronbach, 1951) were calculated for the control group and the ASD parents.

For comparisons of the mean scores of the SF-36 health domains, one-way variance analysis (ANOVA) was performed between each of the three groups of ASD parents and the control parents. Differences obtained were considered statistically significant for a $p$-value less than or equal to 0.05 . Correlation analysis was performed between self-perceived health of ASD mothers and a continuous rating scale of the severity levels of ASD children, provided by CARS. The correlation analysis was performed on greater scale than each individual mother, in order to minimize variations in the SF-36 scores coming from one side the difference between subjectivity of mothers and on the other side from interference between the main concerns and individual and temporary difficulties.

Therefore, we grouped mothers into 9 independent subgroups in relation with the severity levels of the children. In each of these subgroups the severity levels of ASD children was substantially identical and within a range of 3 units of CARS scores. The first subgroup corresponds to the lowest severity, includes children with a CARS scores of $20 \pm 1$. The ninth subgroup corresponds to the highest severity, includes children with a CARS scores of $44 \pm 1$.

Pearson correlation coefficient was calculated between the mean SF-36 scores obtained by mothers and fathers in each 9 subgroups, and the CARS and also ADOS-G scores of ASD children. 


\section{Results}

\subsection{Self-Perceived Health of ASD Parents versus Control Parents}

Mothers and fathers were taken together in the control group and in the three groups of ASD parents. The Cronbach's alpha levels obtained, comprised between 0.7 and 0.9 , showed good internal consistency of the answers given by ASD parents and control parents in each SF-36 scale.

SF-36 scores for the control parents and for the three groups of ASD parents are reported in Table 3 . Table 3 depicts also the decrease in health-related quality of life (HRQOL) of moderate and severe autism parents compared to the control parents.

For the parents of PDD-NOS children, we can observe that the scores, in all of the SF-36 scales, are essentially the same that for the control parents. For the parents of Children with moderate autism symptoms, significant differences $(p<0.05)$ were found with the control parents only in the Mental Health (66 versus 75).

The parents of children with severe autism symptoms have a significantly lower HRQOL than the control parents in five of the eight SF-36 scales and also in the two components summary. The Mental Component Summary revealed large decrements compared to scores for the control group (43 versus 49). Thus, mean reduction for the parents of children with severe autism symptoms was 6 points compared to the control group. This result comes mainly from a substantial HRQOL decrease of the Role Emotional (68 versus 87), the Mental Health (63 versus 75) and the Social Functioning (74 versus 84). The parents of severe autistic children have also a significantly lower mean score of 21 units than the control parents in the Role Physical (69 versus 90).

These results implied that severe autism parents face severe problems with work or other daily activities as a result of emotional problems and also of physical health. However, the Physical Functioning scores remain high for all ASD parents and show no significant difference with the control parents. This result indicates that ASD parents perform all types of activities, including the most trying ones, without limitations.

\subsection{Impact of the Children's Severity on the Self-Perceived Health of ASD Parents}

Correlation coefficient between the self-perceived health of ASD mothers and ASD fathers and the child degree of autistic disorders (scale CARS) are presented in Table 4.

For the SF-36 scales with significant correlation it was possible to use trend curve in order to have better knowledge of the impact of the children's severity on the self-perceived health of parents.

The regression line appears to be the better trend curve to represent the relation between the quality of life of ASD parents and the severity level of the child. In this study, the slope (b) of the regression line corresponds to

Table 3. Mean scores and standard deviations (SD) in the SF-36 scales for control parents and ASD parents.

\begin{tabular}{ccccccc}
\hline & $\begin{array}{c}\text { Control parents } \\
\mathrm{n}=45\end{array}$ & $\begin{array}{c}\text { PDD-NOS } \\
\mathrm{n}=44\end{array}$ & \multicolumn{2}{c}{$\begin{array}{c}\text { Moderate Autism } \\
\mathrm{n}=48\end{array}$} & \multicolumn{2}{c}{$\begin{array}{c}\text { Severe Autism } \\
\mathrm{n}=32\end{array}$} \\
\cline { 2 - 6 } SF-36 Scales & Mean (SD) & Mean (SD) & Mean (SD) & HRQOL Decrease & Mean (SD) & HRQOL Decrease \\
\hline Physical Functioning & $95(10)$ & $91(19)$ & $92(19)$ & 3 & $89(16)^{*}$ & $\mathbf{6}$ \\
Role-Physical & $90(20)$ & $85(25)$ & $89(24)$ & 1 & $69(38)^{*}$ & $\mathbf{2 1}$ \\
Bodily Pain & $85(16)$ & $85(21)$ & $83(24)$ & 2 & $78(28)$ & 7 \\
General Health & $72(14)$ & $73(17)$ & $73(14)$ & 0 & $65(18)$ & 7 \\
Vitality & $61(13)$ & $66(21)$ & $62(19)$ & 0 & $54(26)$ & 7 \\
Social Functioning & $84(18)$ & $86(20)$ & $81(22)$ & 3 & $74(28)^{*}$ & $\mathbf{1 0}$ \\
Role-Emotional & $87(28)$ & $85(30)$ & $85(30)$ & 2 & $68(37)^{*}$ & $\mathbf{1 9}$ \\
Mental Health & $75(13)$ & $75(18)$ & $66(20)^{*}$ & $\mathbf{9}$ & $63(26)^{*}$ & $\mathbf{1 2}$ \\
PCS & $54(6)$ & $53(7)$ & $54(7)$ & 0 & $51(6)^{*}$ & $\mathbf{3}$ \\
MCS & $49(8)$ & $50(11)$ & $46(11)$ & 3 & $43(13)^{*}$ & $\mathbf{6}$
\end{tabular}

PCS physical component summary, MCS mental component summary. ${ }^{*} p<0.05,{ }^{* *} p<0.01$. 
Table 4. Correlation coefficient (r) between the self-perceived health of ASD parents and the severity level of the child. Slope (b) of the regression line.

\begin{tabular}{|c|c|c|c|c|}
\hline \multirow{2}{*}{ SF-36 Scales } & \multicolumn{2}{|c|}{ ASD Mothers } & \multicolumn{2}{|c|}{ ASD Fathers } \\
\hline & Correlation Coefficient (r) & Regression line-Slope (b) & Correlation Coefficient (r) & Regression Line-Slope (b) \\
\hline Physical Functioning & NS & & NS & \\
\hline Role-Physical & $-0.80^{* *}$ & -1.71 & $-0.81^{* *}$ & -1.26 \\
\hline Bodily Pain & $-0.62^{*}$ & -0.97 & NS & \\
\hline General Health & $-0.60^{*}$ & -0.54 & NS & \\
\hline Vitality & $-0.62^{*}$ & -0.79 & $-0.71^{*}$ & -0.88 \\
\hline Social Functioning & $-0.72^{* *}$ & -0.93 & $-0.68^{*}$ & -1.19 \\
\hline Role-Emotional & $-0.82^{* *}$ & -1.83 & $-0.58^{*}$ & -0.73 \\
\hline Mental Health & $-0.80^{* *}$ & -1.09 & $-0.58^{*}$ & -0.73 \\
\hline Physical Component Summary & NS & & NS & \\
\hline Mental Component Summary & $-0.82^{* *}$ & -0.66 & $-0.75^{* *}$ & -0.32 \\
\hline
\end{tabular}

${ }^{*} p<0.05,{ }^{* *} p<0.01$. NS indicates statistically no significant correlation $(p>0.05)$.

the decrease in quality of life of ASD parents, in SF-36 unit, for an increase of 1 unit of the severity level of the child, in the CARS score. Table 4 shows the slopes (b) of the regression line between the quality of life of ASD mothers and ASD fathers and the severity level of the child.

\subsubsection{Standard Deviation between the Mean Scores Obtained in the ASD Mother's Subgroups and Regression Line}

Figure 1 shows a comparison between the mean scores obtained for the Mental Health of ASD mothers, in the nine ASD mother's subgroups, and the regression line.

The regression line allows a good prediction of the impact of the child disorders on the quality of life of the parents. The standard deviation between the predicted scores calculated with the regression line and the mean scores of ASD parent's subgroups is 6 units.

\subsubsection{Mental Component Summary of Control Mothers versus Control Fathers}

The Mental Component Summary scores of control mothers and fathers are shown in Figure 2 by the dotted lines. No significant difference was observed between mothers and fathers of the control group.

\subsubsection{Mental Component Summary of ASD Mothers versus ASD Fathers}

The Mental Component Summary presents significant correlation for ASD mothers $(r=-0.82)$ and ASD fathers ( $r=-0.75$ ), but the slope (b) of the regression line is significantly lower for ASD fathers than for the ASD mothers.

Figure 2 shows a comparison of the Mental Component Summary for ASD mothers and ASD fathers. We can see that the quality of life decreases much more rapidly for mothers than for fathers according to the severity of the child. The mothers of ASD children who have a maximum severity level (45 units, CARS scores) have a significantly lower quality of life of 7 units than the ASD fathers in Mental Component Summary (37 versus 44 ).

We can observe that the Mental Component Summary of ASD father's decreases only for children with a severity level higher than 35 .

\subsubsection{Mental Component Summary of ASD Mothers versus Control Mothers}

Compared to the self perceived health of control mothers, the scores of ASD mothers were significantly different depending on the severity of the children. Maximum decrements were observed for a severity level of 45 units CARS scores, with a decrease of 12 units in the Mental Component Summary. 


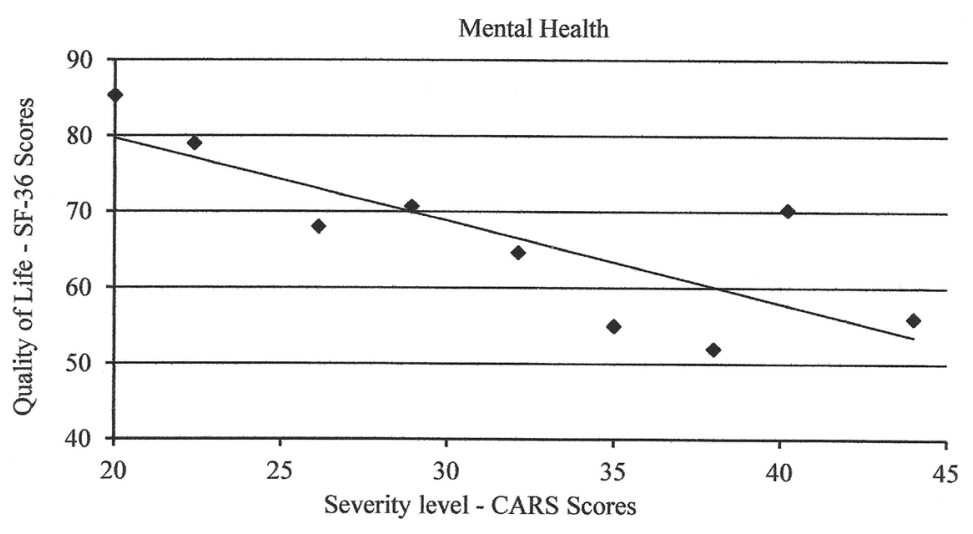

Figure 1. Mental health domain of ASD mothers. Mean scores of the 9 subgroups of mothers (dots) and the regression line, in relation with the severity level of the children.

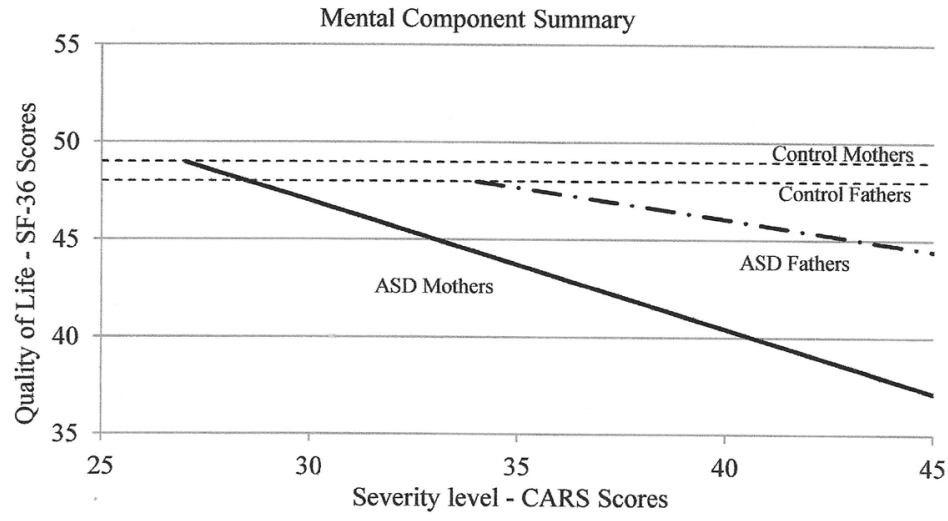

Figure 2. Comparison of the mental component summary for ASD mothers and ASD fathers.

\subsubsection{Health Domains with Significant Correlation with the Severity Level}

For ASD mothers we can observed in Table 4, significant and important correlation $(r=-0.8)$ in three health domains, the Role-Physical, the Role-Emotional, and the Mental Health. ASD fathers show only a single significant correlation coefficient for the Role-Physical.

Figure 3 shows the HRQOL decrease for mothers and fathers, in relation with the scores of the control parents, for the Role-Physical and the Role-Emotional. For ASD mothers, we can clearly observe the impact of the severity level on these two health domains. Difficulty to perform activities is very important for the mother when the severity level of autism is superior to 35 on the CARS scale. For this decrease in the quality of life of the mother, the contributions of mental and physical problems are similar.

For ASD fathers, the impact of the children's severity, on the difficulty to perform daily activities as a result of physical health (Role Physical) is significantly lower than for ASD mothers. Further we can observe that the contribution of mental problems in the difficulty to perform activities (Role Emotional) is two times lower than the contribution of physical problems (Role Physical).

\subsection{Correlation between Autistic Features of ASD Children and the Self-Perceived Health of ASD Mother}

From the point of view of ASD mothers, the four components of autistic features of the children can be considered separately, with more or less important interactions with the quality of life of the mother. The Pearson correlation coefficient between the self-perceived health of ASD mothers and the autistic features of ASD children (ADOS-G) are presented in Table 5. It can be noted that the correlation coefficient is not a causal relationship. 
Table 5. Correlation coefficient (r) between the self-perceived health of ASD mothers (SF-36) and the autistic features of ASD children (ADOS-G).

\begin{tabular}{ccccc}
\hline \multirow{2}{*}{ SF-36 Scales } & \multicolumn{3}{c}{ ADOS-G } & \\
\cline { 2 - 5 } & Social interaction & Communication behaviors & Restrictive behavior & Difficulty in play \\
\hline Physical Functioning & NS & NS & NS & NS \\
Role-Physical & $-0.75^{* *}$ & $-0.63^{*}$ & $-0.66^{*}$ & $-0.67^{*}$ \\
Bodily Pain & $-0.69^{*}$ & $-0.59^{*}$ & NS & NS \\
General Health & $-0.72^{* *}$ & $-0.64^{*}$ & $-0.58^{*}$ & $-0.70^{*}$ \\
Vitality & $-0.62^{*}$ & NS & NS & $-0.55^{*}$ \\
Social Functioning & $-0.65^{*}$ & NS & $-0.56^{*}$ & $-0.76^{* *}$ \\
Role-Emotional & $-0.77^{* *}$ & $-0.63^{*}$ & $-0.72^{* *}$ & $-0.67^{*}$ \\
Mental Health & $-0.77^{* *}$ & $-0.72^{* *}$ & NS & $-0.58^{*}$ \\
\hline Physical Component Summary & NS & NS & $-0.71^{*}$ & NS \\
Mental Component Summary & $-0.76^{* *}$ & $-0.66^{*}$ & $-0.68^{*}$ \\
\hline
\end{tabular}

${ }^{*} p<0.05,{ }^{* *} p<0.01$. NS indicates statistically no significant correlation $(p>0.05)$.

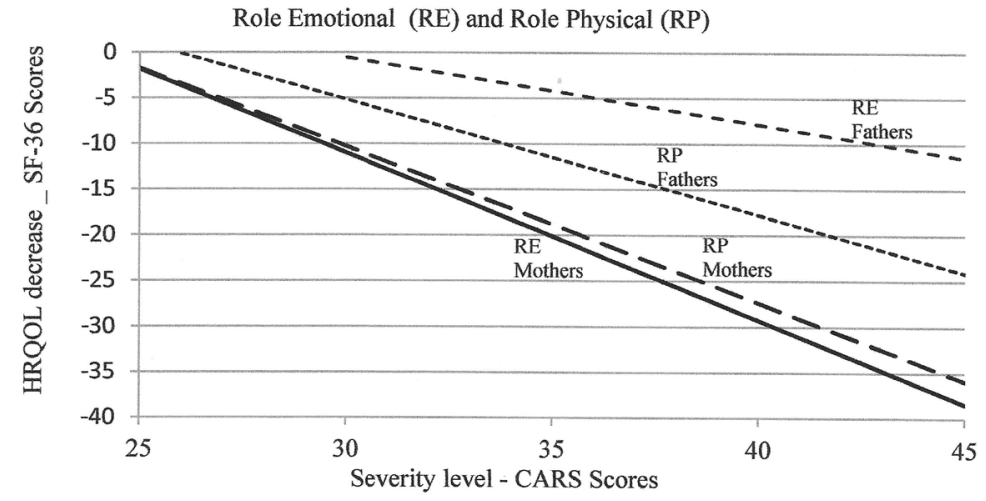

Figure 3. Impact of the severity of ASD children on the Role Emotional and the Role Physical.

Maximum and significant correlations were observed between the social interaction behaviors of the ASD children and four health domains of the mother, Role-Physical, Role-Emotional and General Health and Mental Health. Furthermore we observed high correlation between the social interaction behaviors and the Mental Component Summary of ASD mothers.

All these results suggest that the social interaction behaviors of the children are important for the quality of life of the ASD mothers, particularly in the Mental Health and also the difficulty to perform activities, RolePhysical and the Role-Emotional. The restrictive and repetitive behavior of the children and the difficulty in play are also important for the Role-Emotional.

\section{Discussion}

The results of this study revealed differences in the self-perceived health of ASD parents depending on the child's severity and also according to whether the analysis focuses on fathers and mothers. Comparative analysis between the control parents and three groups of ASD parents show that as the severity of ASD symptoms increase, parent's perception of their own health decreases.

Disorders of children with PDD-NOS, with a score less than 30 in the CARS, have a very weak affect on the quality of life of their parents. No statistically significant differences were observed within the PDD-NOS par- 
ents and the control group. HRQOL of parents of children with moderate autism symptoms decreases slightly in the Mental Health.

Compared to the control group, the parents of children with severe autism symptoms had significantly lower mean scores in two of the health domains (Role-Emotional and Role-Physical). These results implied severe problems with work or other daily activities as a result of emotional problems and also of physical health.

When the self perceived health was calculated separately for mothers and fathers we noted impairment in the Role Emotional and Mental Health significantly greater for ASD mothers than for ASD fathers. Maximum gap between ASD mothers and ASD fathers is observed in the difficulty to perform activities and the feelings of sadness, nervousness and depression. One explanation for this could be an important difference between mothers and fathers in the amount of time spent on the direct daily care of ASD children. These findings confirm previous study reporting that children with a disability have a negative effect on the parents, particularly the mothers (Harvey et al., 1997).

Statistical analysis revealed significant correlations between the severity level of autistic disorder of children and the quality of life of mothers. The Mental Component Summary decreases much more rapidly for mothers than for fathers according to the severity of the children.

We note a very strong relationship between the Mental Health of the mothers and the ASD children, especially in the deficit in social interaction. The Mental Health of ASD mothers decreases in relation to the deficits in developing and maintaining appropriate relationships to developmental level, the apparent lack of interest in people and also the deficits in social-emotional reciprocity.

Deficits in verbal and nonverbal communicative behaviors are also highly correlated with the decrease of Mental Health of ASD mothers. It can be noted that the communication behaviors are necessary factors for the social interaction. Restrictive and repetitive behavior and difficulty in play have less direct impact on the quality of life of ASD mothers. Difficulty to perform activities is very important for the mothers with children affected by severe autism, and the contributions of mental and physical problems are similar.

Relationship between fathers and ASD children is significantly lower than that observed for mothers, except for problems with work or other daily activities as a result of physical health (Role-Physical). The Mental Component Summary of ASD father's decreases only for children with severe autism.

Appropriate psycho-social support should be provided before the health of parents becomes seriously compromised. Evaluation of subsequent interventions in parents of a disabled child is an extremely important issue (Beresford, 1994). Such measures may improve the well-being of autistic children and their families. Additional attention should be focused on the especially vulnerable groups of parents with severe autism children, whose needs for support and help are greater than the needs of other parents. Special attention must be made to the mothers of children with severe autism symptoms.

\section{Conclusion}

In summary, this study has shown impairments in the self-perceived health of mothers of ASD children with severe autism symptoms. First, it seems very important to provide better and more focused support to ASD mothers in order to reduce the difficulty in performing activities. Finding a way to greater involvement of fathers in the care of their children would reduce the pressure supported by mothers.

Secondly, it is necessary to reduce the importance of the stress during the early diagnosis of autism spectrum disorders, especially when the children have a high score in the CARS.

Finally, it seems necessary to improve mental health of mothers, particularly by making easier the process of adaptation with the ASD children.

\section{References}

Abbeduto, L., Seltzer, M. M., Shattuck, P., Krauss, M. W., Orsmond, G., \& Murphy, M. M. (2004). Psychological WellBeing and Coping in Mothers of Youths with Autism, Down Syndrome, or Fragile X Syndrome. American Journal of Mental Retardation, 109, 237-254. http://dx.doi.org/10.1352/0895-8017(2004)109<237:PWACIM>2.0.CO;2

Allik, H., Larsson, J. O., \& Smedje, H. (2006). Health-Related Quality of Life in Parents of School-Age Children with Asperger Syndrome or High-Functioning Autism. Health and Quality of Life Outcomes, 4, 1.

http://dx.doi.org/10.1186/1477-7525-4-1

American Psychiatric Association (2000). Diagnostic and Statistical Manual of Mental Disorders (4th ed.). Washington DC: 
American Psychiatric Association.

American Psychiatric Association (2013). Diagnostic and statistical Manual of Mental Disorders (5th ed.). Washington DC: American Psychiatric Association.

Beckman, P. J. (1995). Comparison of Mothers and Fathers' Perception of Effect of Young Children with and without Disabilities. American Journal of Mental Retardation, 95, 585-595.

Beresford, B. A. (1994). Resources and Strategies: How Parents Cope with the Care of a Disabled Child. Journal of Child Psychology and Psychiatry, 35, 171-209. http://dx.doi.org/10.1111/j.1469-7610.1994.tb01136.x

Coplan, J., \& Jawad, A. F. (2004). Modeling Clinical Outcome of Children with Autistic Spectrum Disorders. Journal of the American Academy of Pediatrics, 116, 117-122. http://dx.doi.org/10.1542/peds.2004-1118

Cronbach, L. J. (1951). Coefficient Alpha and the Internal Structure of Tests. Psychometrika, 16, 297-334. http://dx.doi.org/10.1007/BF02310555

Dyson, L. (1991) Parents of Young Children with Handicaps: Parental Stress and Functioning. American Journal of Mental Retardation, 95, 623-629.

Eaves, R. C., \& Milner, B. (1993). The Criterion-Related Validity of the Childhood Autism Rating Scale and the Autism Behavior Checklist. Journal of Abnormal Child Psychology, 21, 481-491. http://dx.doi.org/10.1007/BF00916315

Freeman, N. L., Perry, A., \& Factor, D. C. (1991). Child Behaviours as Stressors: Replicating and Extending the Use of the CARS as a Measure of Stress: A Research Note. Journal of Child Psychology and Psychiatry, 32, 1025-1030. http://dx.doi.org/10.1111/j.1469-7610.1991.tb01927.x

Harvey, J. M., O’Callaghan, M. J., \& Vines, B. (1997). Prevalence of Maternal Depression and Its Relationship to ADL Skills in Children with Developmental Delay. Journal of Pediatrics and Child Health, 33, 42-46.

http://dx.doi.org/10.1111/j.1440-1754.1997.tb00989.x

Hastings, R. P. (2002). Parental Stress and Behaviour Problems of Children with Developmental Disability. Journal of Intellectual Disability Research, 27, 149-160. http://dx.doi.org/10.1080/1366825021000008657

Herring, S., Gray, K., Taffe, J., Tonge, B., Sweeney, D., \& Einfeld, S. (2006). Behavior and Emotional Problems in Toddlers with Pervasive Developmental Disorders and Developmental Delay: Associations with Parental Mental Health and Family Functioning. Journal of Intellectual Disability Research, 50, 874-882.

Leplège, A., Ecosse, E., Verdier, A., \& Perneger, T. V. (1998). The French SF-36 Health Survey: Translation, Cultural Adaptation and Preliminary Psychometric Evaluation. Journal of Clinical Epidemiology, 51, 1013-1023. http://dx.doi.org/10.1016/S0895-4356(98)00093-6

Lord, C., Risi, S., Lambrecht, L., Cook, E. H., Leventhal, B. L., DiLavore, P. C. et al. (2000). The Autism Diagnostic Observation Schedule Generic: A Standard Measure of Social and Communicative Deficits Associated with the Spectrum of Autism. Journal of Autism and Developmental Disorders, 30, 205-223. http://dx.doi.org/10.1023/A:1005592401947

Magyar, C. I., \& Pandolfi, V. (2007). Factor Structure Evaluation of the Childhood Autism Rating Scale. Journal of Autism and Developmental Disorders, 37, 1787-1794. http://dx.doi.org/10.1007/s10803-006-0313-9

McHorney, C. A., Ware Jr., J. E., \& Raczek, A. E. (1993). The MOS 36-Item Short-Form Health Survey (SF-36): II. Psychometrics and Clinical Tests of Validity in Measuring Physical and Mental Health Constructs. Medical Care, 31, 247263. http://dx.doi.org/10.1097/00005650-199303000-00006

Olsson, M. B., \& Hwang, C. P. (2001). Depression in Mothers and Fathers of Children with Intellectual Disability. Journal of Intellectual Disability Research, 45, 535-543. http://dx.doi.org/10.1046/j.1365-2788.2001.00372.x

Perry, A., Condillac, R. A., Freeman, N. L., Dunn-Geier, J., \& Belair, J. (2005). Multi-Site Study of the Childhood Autism Rating Scale (CARS) in Five Clinical Groups of Young Children. Journal of Autism and Developmental Disorders, 35, 625-634. http://dx.doi.org/10.1007/s10803-005-0006-9

Rutter, M., Le Coutteur, A., \& Lord, C. (2003). ADI-R: The Autism Diagnostic Interview-Revised. Los Angeles, CA: Western Psychological Services.

Schopler, E., Reichler, R. J., De Vellis, R. F., \& Daly, K. (1980). Toward Objective Classification of Childhood Autism: Childhood Autism Rating Scale (CARS). Journal of Autism and Developmental Disorders, 10, 91-103. http://dx.doi.org/10.1007/BF02408436

Schopler, E., Reichler, R. J., \& Renner, B. R. (1988). The Childhood Autism Rating Scale. Los Angeles, CA: Western Psychological Services.

Schopler, E., Van Bourgondien, M. E., Wellman, G. J., \& Love, S. R. (2010). The Childhood Autism Rating Scale (2nd ed.) (CARS2). Los Angeles, CA: Western Psychological Services.

Shu, B. C. (2009). Quality of Life of Family Caregivers of Children with Autism: The Mother's Perspective. Autism, 13, 8191. http://dx.doi.org/10.1177/1362361307098517 
Trute, B. (1995). Gender Differences in the Psychological Adjustment of Parents of Young, Developmentally Disabled Children. Journal of Child Psychology and Psychiatry, 36, 1225-1242.

Ware, J. E., Kosinski, M., \& Keller, S. D. (1994). SF-36 Physical and Mental Health Summary Scales: A User's Manual. Boston, MA: The Health Institute, New England Medical Center.

Wechsler, D. (1992). Wechsler Intelligence Scale for Children (3rd ed.). London: Psychological Corporation.

Wechsler, D. (2002). Wechsler Preschool and Primary Scale of Intelligence (3rd ed.). San Antonio, TX: Psychological Corporation.

WHOQOL Group (1995). The World Health Organization Quality of Life Assessment (WHOQOL): Position Paper from the World Health Organization. Social Science \& Medicine, 41, 1403-1409. http://dx.doi.org/10.1016/0277-9536(95)00112-K

Zablotsky, B., Anderson, C., \& Law, P. (2012). The Association between Child Autism Symptomatology, Maternal Quality of Life, and Risk of Depression. Journal of Autism and Developmental Disorders, 10, 91-103. 
Scientific Research Publishing (SCIRP) is one of the largest Open Access journal publishers. It is currently publishing more than 200 open access, online, peer-reviewed journals covering a wide range of academic disciplines. SCIRP serves the worldwide academic communities and contributes to the progress and application of science with its publication.

Other selected journals from SCIRP are listed as below. Submit your manuscript to us via either submit@scirp.org or Online Submission Portal.
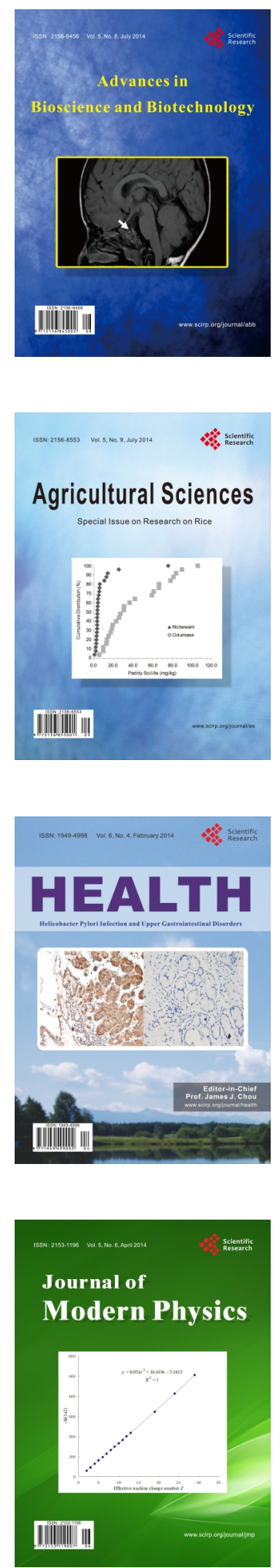
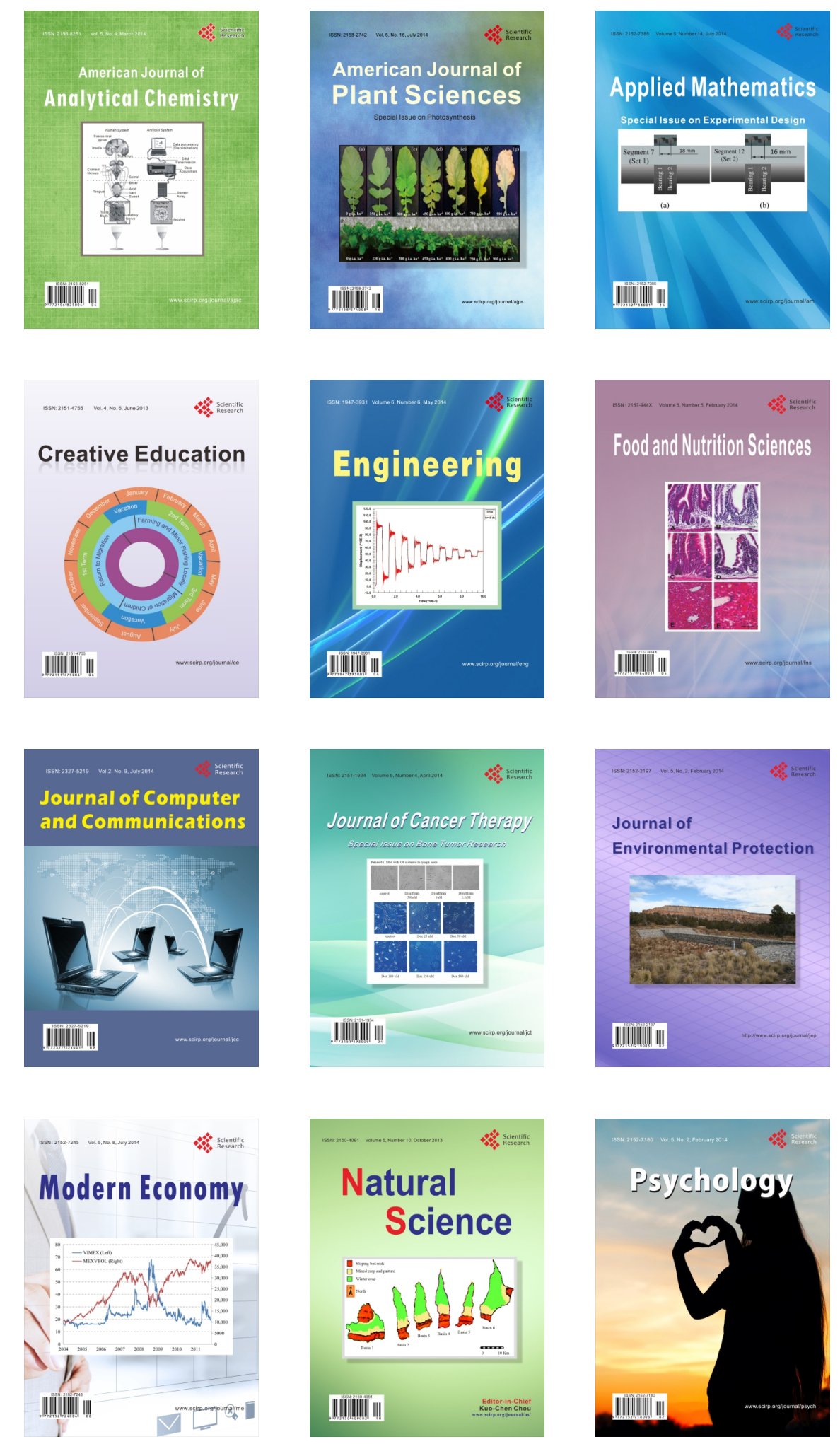